\title{
Hollow core photonic crystal fiber-assisted Raman spectroscopy as a tool for the detection of Alzheimer's disease biomarkers
}

\author{
Pinkie J. Eravuchira, ${ }^{\text {a, } *, \dagger}$ Martina Banchelli, ${ }^{\text {b }}$ Cristiano D’Andrea, ${ }^{\text {b }}$ \\ Marella de Angelis, ${ }^{b}$ Paolo Matteini, ${ }^{b, *}$ and Israel Gannot ${ }^{a, c, *}$ \\ ${ }^{a}$ Tel Aviv University, Department of Biomedical Engineering, Faculty of Engineering, \\ Tel Aviv, Israel \\ bInstitute of Applied Physics "NelloCarrara," National Research Council, Sesto Fiorentino, Italy \\ 'Johns Hopkins University, Department of Electrical and Computer Engineering, Baltimore, \\ Maryland, United States
}

\begin{abstract}
Significance: Alzheimer's disease (AD) is an irreversible and progressive disorder that damages brain cells and impairs the cognitive abilities of the affected. Developing a sensitive and cost-effective method to detect Alzheimer's biomarkers appears vital in both a diagnostic and therapeutic perspective.

Aim: Our goal is to develop a sensitive and reliable tool for detection of amyloid $\beta$ (1-42) peptide (A $\beta 42)$, a major AD biomarker, using fiber-enhanced Raman spectroscopy (FERS).

Approach: A hollow core photonic crystal fiber (HCPCF) was integrated with a conventional Raman spectroscopic setup to perform FERS measurements. FERS was then coupled with surface-enhanced Raman spectroscopy (SERS) to further amplify the Raman signal thanks to a combined FERS-SERS assay.

Results: A minimum 20-fold enhancement of the Raman signal of $\mathrm{A} \beta 42$ as compared to a conventional Raman spectroscopy scheme was observed using the HCPCF-based light delivery system. The signal was further boosted by decorating the fiber core with gold bipyramids generating an additional SERS effect, resulting in an overall 200 times amplification.

Conclusions: The results demonstrate that the use of an HCPCF-based platform can provide sharp and intense Raman signals of $\mathrm{A} \beta 42$, in turn paving the way toward the development of a sensitive label-free detection tool for early diagnosis of $\mathrm{AD}$.

(C) The Authors. Published by SPIE under a Creative Commons Attribution 4.0 Unported License. Distribution or reproduction of this work in whole or in part requires full attribution of the original publication, including its DOI. [DOI: 10.1117/1.JBO.25.7.077001]
\end{abstract}

Keywords: Alzheimer's; amyloid $\boldsymbol{\beta}$-peptide; fiber-enhanced Raman spectroscopy; liquid biopsy; surface-enhanced Raman spectroscopy.

Paper 200063RR received Mar. 11, 2020; accepted for publication Jun. 11, 2020; published online Jul. 2, 2020.

\section{Introduction}

Alzheimer's disease (AD) is a chronic, progressive, neurodegenerative disorder that affects several million of people worldwide. ${ }^{1-4} \mathrm{AD}$ is considered as one of the leading causes of dementia, and it is ranked as the fifth major cause of death. $\mathrm{AD}$ is pathologically characterized by the deposition of extracellular plaques composed of amyloid $\beta$-peptide $(\mathrm{A} \beta)$ and the aggregation of tau protein as intracellular neurofibrillary tangles in the brain. ${ }^{5-10}$ Specifically, the entorhinalcortex and hippocampus, which are brain areas dealing with cognitive abilities, are critically

\footnotetext{
*Address all correspondence to Pinkie J. Eravuchira, E-mail: pinkiejacob@gmail.com; Paolo Matteini, E-mail: p.matteini@ifac.cnr.it; Israel Gannot, E-mail: gannoti@tauex.tau.ac.il

${ }^{\dagger}$ Present address: Christ (Deemed to be University), Department of Physics and Electronics, Bangalore, India
} 
affected by the deposition of plaques and tangles in such a way that cognitive disabilities, including memory impairment, difficulties with reasoning and solving problems, and challenges with time and space, are among the prime distinctive symptoms of AD. The current antemortem $\mathrm{AD}$ diagnosis is based on monitoring the mental decline and on neuropsychological and laboratory tests. Nevertheless, the conclusive diagnosis of $\mathrm{AD}$ is still challenging requiring an autopsy substantiation of disease pathology.

Molecular biomarkers are explicit indicators of a specific health and disease state, and their detection is crucial for diagnosis, monitoring of disease progression, and treatment. Protein species such as $\mathrm{A} \beta$ and tau protein are associated with $\mathrm{AD}$ and recognized as main biomarkers of AD. Studies evidenced that biochemical changes leading to progressive accumulation of such biomarkers begin even 15 to 20 years before the first symptom appears. Although as of now there is no definitive cure for $\mathrm{AD}$, several medications that may slow down the progress of $\mathrm{AD}$ are already available in the market. ${ }^{11,12}$ Therefore, a presymptomatic diagnosis of $\mathrm{AD}$ is fundamental as it would aid to maintain normal brain activity. Tremendous efforts are being made to develop effective and ultrasensitive methods for the detection of AD biomarkers. Amyloid positron emission tomography (PET) represents a powerful and efficient technique for imaging of $\mathrm{A} \beta$ in the brain at an early stage of the disease progression. ${ }^{13-16}$ Nonetheless, high costs, limited accessibility, and health hazards restrain this technique as a gold standard for AD diagnosis. On the other hand, detection of trace amounts of $\mathrm{A} \beta$ and tau protein shed into the cerebrospinal fluid (CSF) or other accessible biofluids is now recognized as a potential alternative for early AD diagnosis. ${ }^{17,18}$ On this basis, developing a cost-effective, reliable, and sensitive technique that allows for sensitive detection of AD biomarkers at an early stage will be a huge advancement in the field of AD diagnosis and treatment. Numerous techniques such as liquid chromatography, enzyme-linked immunosorbent assay (ELISA), mass spectrometry, flow cytometry, electrochemical sensing, surface plasmon resonance, fluorescence, and Raman spectroscopy are being studied and tested for the detection of AD biomarkers. ${ }^{6,19-27}$ Among the optical methods, Raman spectroscopy and its related techniques are being demonstrated as a reliable tool providing labelfree fingerprint information from biomolecules including $\mathrm{AD}$ biomarkers and misfolded protein species associated with neurodegenerative conditions. ${ }^{28-32}$ High chemical specificity, noninvasiveness, reproducibility, and rapidness make present Raman-based methods a promising option for the detection of $\mathrm{AD} .{ }^{33-35}$ Nevertheless, the low efficiency of the Raman scattering process and high-fluorescence background limit this technique for the detection of biomolecules, especially when the concentration of the analyte is reduced. The weak Raman scattering can be compensated by either increasing the excitation laser power or by increasing the number of analyte molecules that interact with laser light. However, increasing the laser power is not an ideal solution, especially for heat-sensitive molecules, as are most of the biomolecules. Fiber-enhanced Raman spectroscopy (FERS) can address the above limitations by increasing the interaction between analyte molecules and the excitation laser. FERS is a method that integrates a microstructured optical fiber with a conventional Raman spectroscopic system and has gained remarkable attention as a detection technique for applications ranging from biosensing to health monitoring. ${ }^{34-46}$ Among the microstructured optical fibers, hollow core photonic crystal fiber (HCPCF) has proved to be a potential detection tool, especially for the analysis of trace amounts of analyte molecules. HCPCF is a novel microstructured photonic crystal fiber that has a central hollow core surrounded by a periodic arrangement of air holes. An incident laser of a definite wavelength gets confined into the fiber core due to the photonic band-gap effect of the periodic array cladding and the light spreads through the fiber core with very low attenuation. FERS based on HCPCF has proven convenient for a broad range of applications dealing with the detection of liquid and gaseous samples. ${ }^{36-38}$ In this case, the analyte is collected inside the fiber holes, i.e., making HCPCF act as a miniaturized sample container, whereas an increased interaction between laser light and analyte molecules is achieved within the HCPCF core. Compared to a conventional Raman spectroscopic approach, Raman scattering is highly enhanced due to the unique FERS geometry, which, in turn, improves the detection sensitivity. Additionally, a stable light coupling can be achieved using HCPCF, as the transmission spectrum of HCPCF remains unaffected even with the bending of the optical fiber.

Exploiting the optical properties of the HCPCF, we developed an ultrasensitive and label-free sensing system based on FERS for the detection of A $\beta$. For this purpose, an HCPCF-based light 
delivery system was properly assembled for the Raman measurements. In addition, to study the possibility to further increase the signal, the FERS system was integrated with surface-enhanced Raman spectroscopy (SERS), as extra expedient to circumvent the sensitivity limitations of Raman scattering. Low sampling volume, compact size, sensitivity, rapidness, and costeffectiveness make the proposed label-free platform a promising detection tool for the early diagnosis of AD pathology.

\section{Methods}

\subsection{Reagents and Chemicals}

Myoglobin $(\mathrm{Mb})$ from horse skeletal muscle and Amyloid $\beta(1-42)$ peptide $(\mathrm{A} \beta 42)$ were purchased from Sigma-Aldrich and used as received. A $\beta 42$ solution was prepared by dissolving A $\beta 42$ powder in $50 \mathrm{mM} \mathrm{NaOH}$ and finally diluting in phosphate-buffered saline (PBS) at the desired final concentration.

For SERS experiments, a colloidal solution of cetrimonium bromide-capped gold nanobipyramids (AuBPs, $1 \mathrm{mg} / \mathrm{ml}$ in water) having a diameter and length of 35 and $105 \mathrm{~nm}$, respectively, was used (Nanoseedz, Hong Kong). Milli-Q water was used throughout our experiments. Fresh solutions of biomolecules were considered for the measurements.

\subsection{Optical Fiber-Based Light Delivery System for Raman Measurements}

An HCPCF-based light delivery system was assembled for carrying out the Raman measurements. The optical setup consisted of a commercial confocal Raman microspectrometer (Labram HR, Horiba Jobin-Yvon, Japan) coupled with a homemade fiber holder that holds the HCPCF (Fig. 1). The excitation laser produced by a solid-state diode laser source emitting at $532 \mathrm{~nm}$ was focused into the fiber core using a $50 \times$ long working distance microscope objective (NA 0.55, Olympus). The Raman scattered light from the sample propagated back through the fiber and was collected in a backscattering geometry using the same objective, was analyzed by a diffraction grating (600 grooves $/ \mathrm{mm}$ ), and acquired by a charged coupled device (Synapse CCD Detector) embedded in the spectrometer.

All experiments were performed with an incident power measured on the sample of $5 \mathrm{~mW}$, whereas the spectral acquisition times for ethanol, $\mathrm{Mb}$, and $\mathrm{A} / 442$ were 5,10 , and $15 \mathrm{~s}$,

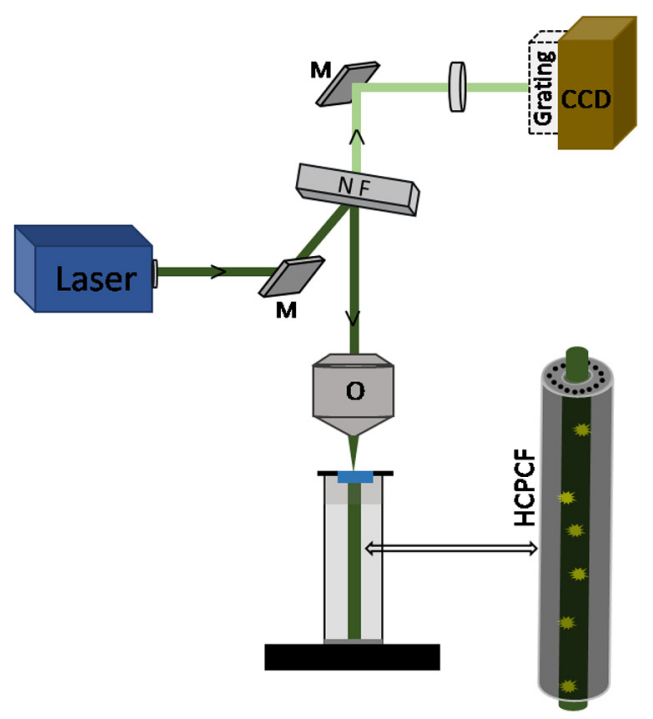

Fig. 1 Schematic of the fiber-enhanced Raman spectroscopic setup. (M, mirror; NF, notch filter; and $\mathrm{O}$, microscopic objective). 
respectively. Before measurements, the spectroscopic system was calibrated on the $520.8 \mathrm{~cm}^{-1}$ Raman peak of a silicon wafer.

Commercial hollow core crystal fibers (Model HC-1060, Thorlabs) were used in FERS experiments. Both ends of a 5-cm-long fiber were first cleaved, then the fiber was meticulously vertically aligned to the microscope objective using a fiber holder mounted on an $X Y Z$ stage. The chamber of the fiber holder was filled with the sample solution $(30 \mu \mathrm{l})$, which was then allowed to flow down through the microcavities of the fiber for $2 \mathrm{~min}$ before the measurements. Photodegradation of the analytes during Raman spectra acquisition was cautiously prevented by assuring a smooth continuous flow of analyte solution through the fiber during the measurements. This was achieved by pumping the analyte solution using a syringe pump in to the sample holder at a regular interval of time. Control measurements were carried out by direct Raman sampling, in which the HCPCF was replaced with an open cuvette filled with the analyte solution and the laser was focused onto the exposed solution in the open cuvette.

\section{Results and Discussion}

\subsection{Fiber-Enhanced Raman Sensing}

In order to compare the vibrational signal response produced by our FERS system with that obtained by a conventional Raman spectroscopy setup, Raman measurements were carried out both with the analyte solution inside the HCPCF and with a conventional direct sampling method. The HCPCF (Fig. 2) used in the experiments shows a core diameter of $10 \mu \mathrm{m}$, and its pitch diameter is about $2.6 \mu \mathrm{m}$. The microstructured air holey cladding region is periodically arranged around the central core with a total dimension of about $50 \mu \mathrm{m}$ with an air filling fraction of $>90 \%$.

The background Raman spectrum of the optical fiber was preliminarily measured in order to assess the absence of any interference with the Raman spectra of the analytes. We verified that once the HCPCF is filled with water, due to a change in the refractive index, its transmission spectrum gets shifted from NIR (centerd at $1060 \mathrm{~nm}$ ) to the visible range with maximum transmission in the region from 500 to $900 \mathrm{~nm}^{36}$ (Fig. 3).

\subsection{FERS of Ethanol and Myoglobin}

Initially, the Raman spectrum of a $25 \%$ (v/v) ethanol-water solution was recorded. Figure 4(a) shows a comparison between the Raman spectral profiles of ethanol as obtained by direct and HCPCF-mediated sampling. The Raman peak at $880 \mathrm{~cm}^{-1}$, which is attributed to the skeletal CCO symmetric stretching ${ }^{36,47}$ of ethanol, appears in both spectra. Nevertheless, compared to the sample measured inside the HCPCF, its peak intensity collected by conventional sampling

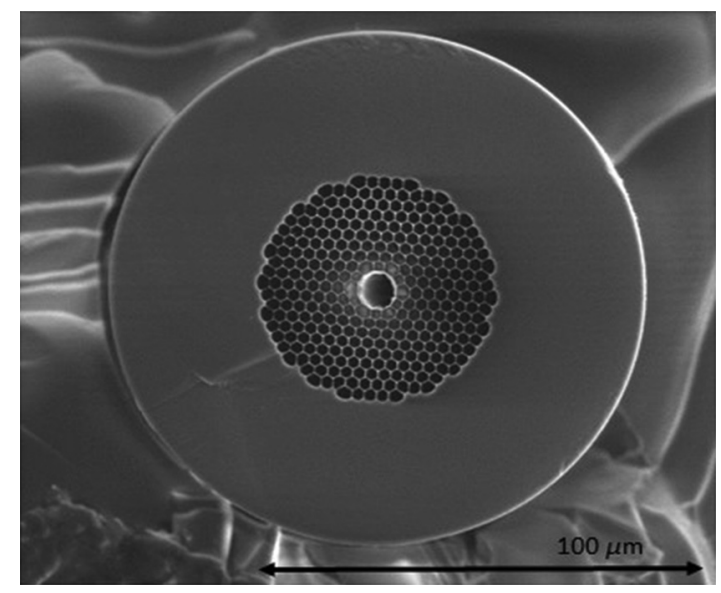

Fig. 2 SEM image of the cleaved end of the HCPCF used in the experiments. 


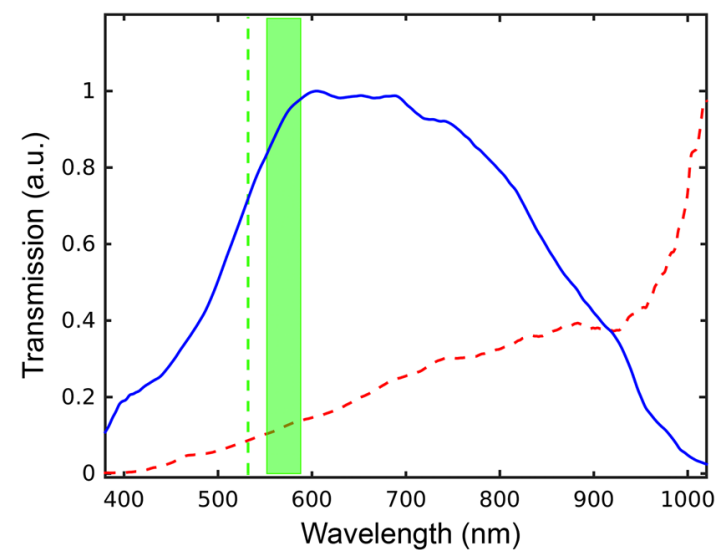

Fig. 3 Transmission spectra of the HCPCF filled with air (red dashed line) and with water (blue continuous line). The excitation line at $532 \mathrm{~nm}$ (green dashed line) and the spectral range (green band) considered in our Raman experiments are also shown.
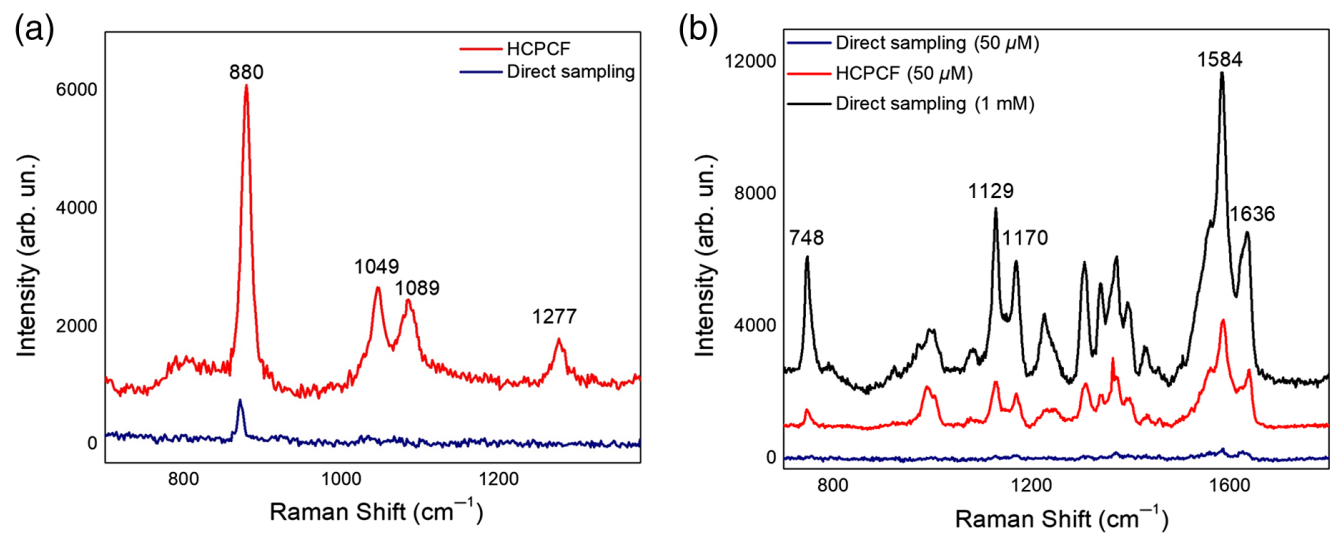

Fig. 4 Comparison between Raman spectra acquired by direct sampling (blue, black lines) and using the HCPCF setup (red lines) of (a) a $25 \%$ ethanol solution and (b) Mb at $1 \mathrm{mM}$ (black lines) and $50 \mu \mathrm{M}$ (blue, red lines).

appeared 10-fold lower. Moreover, the Raman peaks at 1049, 1089, and $1277 \mathrm{~cm}^{-1}$, which are assigned to skeletal CCO deformations, CO stretching, and deformation wagging of ethanol, respectively, were only detected in the sample measured in HCPCF. Subsequently, in order to test the system performances for biomolecule analysis, we examined the vibrational spectra of $\mathrm{Mb}$, here taken as a model of a biomolecule extensively investigated in Raman studies ${ }^{48,49}$ [Fig. 4(b)]. Prominent vibrational peaks at 1129, 1170, 1306, 1372, 1584, and $1636 \mathrm{~cm}^{-1}$, which are attributed to $v 22, v 30, v 21, v 4, v 19$, and $v 10$ of the heme group, respectively, ${ }^{48,50}$ were observed by direct sampling of a highly concentrated $(1 \mathrm{mM})$ protein solution. Further measurements were carried out by decreasing the protein concentration down to $50 \mu \mathrm{M}$. Similar to the case of ethanol, intense Raman signals were collected by FERS, while most of them were undistinguishable or noisy in the spectrum obtained by the conventional Raman scheme. These results offered us a first test bench proving the efficiency of our FERS-based setup.

\subsection{FERS of $\mathrm{A} \beta 42$}

Once we clarified the potential offered by FERS with HCPCF, we verified its efficacy in the Raman detection of $\mathrm{A} \beta 42$. Initially, measurements of $\mathrm{A} \beta$ with a concentration of $100 \mu \mathrm{g} / \mathrm{ml}$ were performed both under direct sampling and by the HCPCF-based setup [Fig. 5(a)]. Characteristic Raman modes of $\mathrm{A} \beta$ peaking at 830/850, 1003, 1457, and $1660 \mathrm{~cm}^{-1}$ can be 

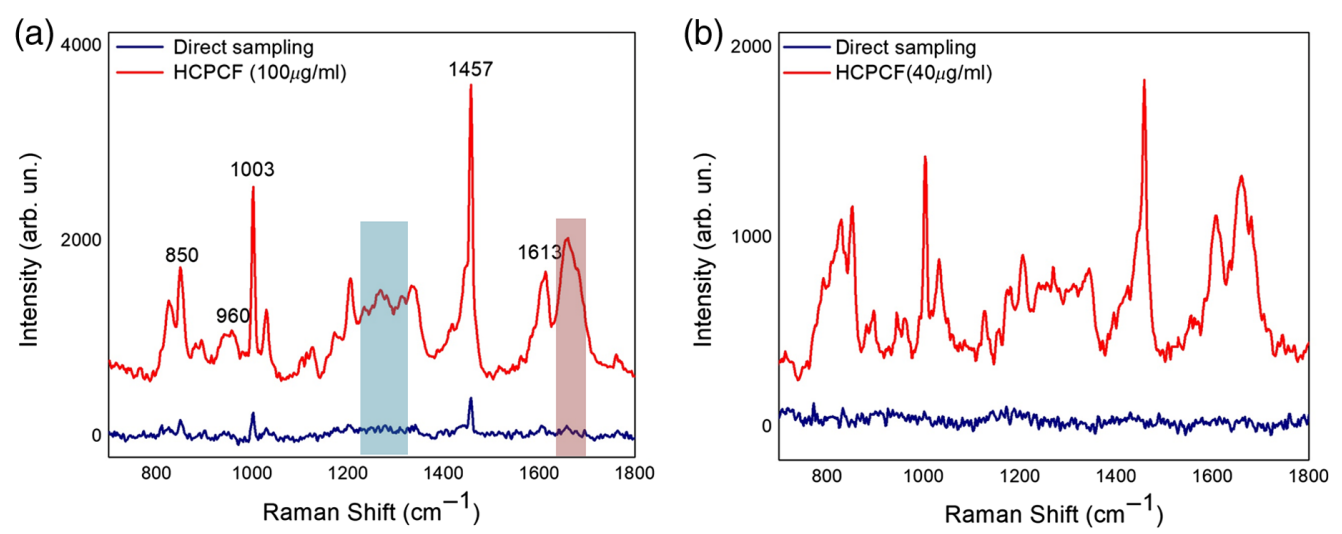

Fig. 5 Raman spectra of $\mathrm{A} \beta 42$ acquired by conventional Raman spectroscopy (blue lines) and with an HCPCF (red lines): (a) $100 \mu \mathrm{g} / \mathrm{ml}$ and (b) $40 \mu \mathrm{g} / \mathrm{ml}$. Shaded regions identify amide I (red) and amide III (blue) bands of $\mathrm{A} \beta$.

identified by both approaches and ascribed to the Fermi doublet of Tyr, the aromatic Phe ring breathing, $\mathrm{CH}_{2}$ scissoring vibrations, and the amide I region, respectively ${ }^{44-48}$ (Table 1). Additional peaks were detected in the FERS spectrum ascribed to the backbone CCN stretching vibration $\left(960 \mathrm{~cm}^{-1}\right)$, the amide III band (1230 to $\left.1300 \mathrm{~cm}^{-1}\right)$, and the aromatic Tyr ring stretching $\left(1613 \mathrm{~cm}^{-1}\right)$. In accordance with previous results on ethanol and $\mathrm{Mb}$, the spectral features of $\mathrm{A} \beta$ measured using HCPCF appeared sharp and intense in such a way that a signal enhancement of $\sim 20$-fold at the minimum was observed as compared to the direct sampling modality. Nonetheless, apart from some exceptions (e.g., amide I, amide III, the $850-\mathrm{cm}^{-1}$ band of Tyr, and the $1003-\mathrm{cm}^{-1}$ band of Phe), the peaks of the $\mathrm{A} \beta$ solution measured by conventional Raman showed a low signal-to-noise ratio or were mostly imperceptible. These outcomes validated the potential of FERS with HCPCF for efficient detection of $\mathrm{A} \beta$.

Afterward, we spent effort in demonstrating the sensitivity of our FERS setup upon decreasing the $\mathrm{A} \beta$ concentration. Successive FERS measurements were performed on a $40-\mu \mathrm{g} / \mathrm{ml}$ solution of $\mathrm{A} \beta$ [Fig. 5(b)]. In this case, the vibrational spectra measured with the FERS system

Table 1 Assignments of main Raman peaks of $\mathrm{A} \beta 42 .{ }^{49}$

\begin{tabular}{ll}
\hline \hline Peak position $\left(\mathrm{cm}^{-1}\right)$ & Mode assignment \\
\hline 830 & Tyr \\
850 & Tyr \\
960 & CC str \\
1003 & Phe \\
1030 & Phe \\
1130 & CN str \\
1205 to 1210 & Phe/Tyr \\
1230 to 1270 & Amide III \\
1455 to 1465 & CH def \\
1530 & His/amide II \\
1613 & Tyr \\
1660 to 1690 & Amide I \\
\hline \hline
\end{tabular}




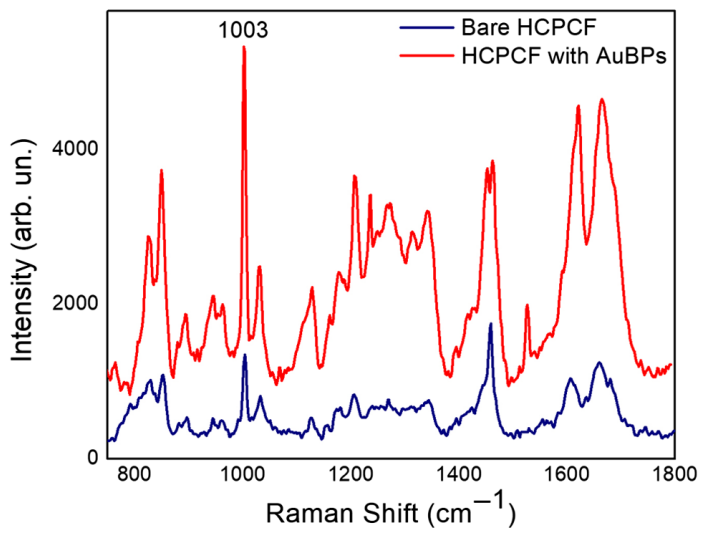

Fig. 6 Raman spectra of $40 \mu \mathrm{g} / \mathrm{ml} \mathrm{A} \beta 42$ achieved with a bare HCPCF (blue line) and with the AuBPs-coated HCPCF (red line).

appeared strongly enhanced compared to those obtained by conventional Raman, which were noisy without any distinct Raman peaks detected.

Aimed at further improving the optical performances of our setup, we integrated the FERS system with AuBPs within the fiber core to take advantage of a supplementary SERS effect. The choice of using nonspherical nanoparticles here is motivated because of their well-established superior SERS efficiency generated by effective SERS hot spots at the sharp ends. ${ }^{51,52}$ AuBPs were adhered to the fiber core by electrostatic-mediated surface immobilization between positively charged nanoparticles and the negative surface charge of the inner silica core of the fiber. In short, the nanoparticle solution was allowed to enter into the fiber core by dipping the HCPCF in $2 \mathrm{ml}$ of particle solution for about $5 \mathrm{~min}$. The fiber was then dried at $40^{\circ} \mathrm{C}$ in an oven for $5 \mathrm{~min}$ to remove the solvent and extensively rinsed with Milli-Q water to remove unbound nanoparticles. The $\mathrm{A} \beta$ solution $(40 \mu \mathrm{g} / \mathrm{ml})$ was then allowed to flow through the particle-coated fiber core as in the previous measurements. Figure 6 depicts a comparison between spectra obtained by SERS-active HCPCF and bare HCPCF. We demonstrated that an additional $~ 10$ times signal enhancement could be attained using the AuBPs-functionalized fiber. All the prominent characteristic Raman peaks of $\mathrm{A} \beta$ were identified in the SERS spectrum, suggesting that the native structure of the inspected species was almost preserved.$^{53}$ The appearance of prevailing peaks as, e.g., Tyr modes at 830,850 , and $1613 \mathrm{~cm}^{-1}$, Phe modes at 1003 and $1205 \mathrm{~cm}^{-1}$, as well as $\mathrm{C}-\mathrm{N}$ vibrational modes from aminoterminated amino acids within the 1080 -to $1150-\mathrm{cm}^{-1}$ region may represent an indication of the spatial proximity of these residues to the Au surface. ${ }^{49}$ An overall signal amplification of $\sim 200$ times can be finally estimated as provided by both the use of a HCPCF and its integration with AuBPs within our Raman setup.

This work represents a proof-of-concept demonstration of an effective Raman detection system of $\mathrm{A} \beta 42$ showing high sensitivity combined with well-resolved and intense spectral A $\beta 42$ features. Thanks to the above characteristics, the proposed FERS-SERS method proves suitable for future implementations aimed at extending the limit of detection to match physiological requirements. A $\beta 42$ in the CSF of healthy humans ranges around $1.2 \pm 0.4 \mathrm{ng} / \mathrm{ml}$ and decreases in the case of AD patients. ${ }^{54,55}$ Future efforts will be spent to optimize the system by a comparison among different plasmonic nanoparticles, a tight control over their density, as well as their possible functionalization with biorecognition elements.

\section{Conclusion}

We demonstrated the advantages in supplying a standard Raman setup with a fiber-based Raman spectroscopic system for the analysis of molecules of biological and biomedical interest, including $\mathrm{A} \beta$ peptide as an established biomarker of $\mathrm{AD}$. The measurements were performed using a HCPCF with a laser excitation wavelength of $532 \mathrm{~nm}$. Increased interaction between the analyte and the guided laser excitation light as obtained inside an HCPCF leads to a flexible, reliable, and sensitive tool for the enhancement of weak Raman signals, such as those usually encountered 
with biomolecules. The results attested that, compared to the conventional direct sampling method, the FERS approach can generate a $\geq 20$ times amplified signal from protein species. Furthermore, by equipping the HCPCF with plasmonic nanoparticles, the Raman signal can undergo an overall $\sim 200$ times enhancement as a result of a supplementary SERS effect. Finally, advantages such as low sampling volume, the possibility of in situ measurements, flexibility, portability, and cost-effectiveness depict the proposed platform as particularly attractive in view of a preclinical or clinical detection of $\mathrm{A} \beta$, paving the way toward an early detection of Alzheimer's. Further studies will be aimed at verifying the potential of different metal nanoparticles and their tailored assembly inside the fiber core in further implementing the platform when using reduced concentration values of the analyte.

\section{Disclosures}

The authors declare no financial or commercial conflicts of interest.

\section{Acknowledgments}

Authors acknowledge the support from the European Community, the Israeli Ministry of Health, University and Research of Italy (MIUR) through the ERANET EuroNanoMed III SPEEDY Project under Grants No. 00370311000 and No. ID221. We acknowledge the previous SPIE Proceedings publications.

\section{References}

1. G. Cornutiu, "The epidemiological scale of Alzheimer's disease," J. Clin. Med. Res. 7(9), 657-666 (2015).

2. F. A. Etindele Sosso, O. Nakamura, and M. Nakamura, "Epidemiology of Alzheimer's disease: comparison between Africa and South America," J. Neurol. Neurosci. 8(4), 2015-2017 (2017).

3. J. Gaugler et al., "2016 Alzheimer's disease facts and figures," Alzheimer's Dement. 12(4), 459-509 (2016).

4. B. Duthey, Alzheimer Disease and Other Dementias, pp. 1-77, World Health Organization (2013).

5. D. Galasko, "Expanding the repertoire of biomarkers for Alzheimer's disease: targeted and non-targeted approaches," Front. Neurol. 6(Dec.), 1-13 (2015).

6. C. Humpel, "Identifying and validating biomarkers for Alzheimer's disease," Trends Biotechnol. 29(1), 26-32 (2011).

7. N. El Kadmiri et al., "Biomarkers for Alzheimer disease: classical and novel candidates' review," Neuroscience 370, 181-190 (2018).

8. L. F. Lue, A. Guerra, and D. G. Walker, "Amyloid beta and Tau as Alzheimer's disease blood biomarkers: promise from new technologies," Neurol. Ther. 6(s1), 25-36 (2017).

9. S. Y. Ow and D. E. Dunstan, "A brief overview of amyloids and Alzheimer's disease," Protein Sci. 23(10), 1315-1331 (2014).

10. F. Chiti and C. M. Dobson, "Protein misfolding, amyloid formation, and human disease: a summary of progress over the last decade," Anпи. Rev. Biochem. 86(1), 27-68 (2017).

11. J. L. Cummings, G. Tong, and C. Ballard, "Treatment combinations for Alzheimer's disease: current and future pharmacotherapy options.," J. Alzheimers. Dis. 67(3), 779-794 (2019).

12. N. Kristina et al., "Alzheimer's disease: current and potential new treatments," U.S. Pharm. 44(1), 20-23 (2019).

13. C. Marcus, E. Mena, and R. M. Subramaniam, "Brain PET in the diagnosis of Alzheimer's disease," Clin. Nucl. Med. 39(10), e413-e426 (2014).

14. R. Vandenberghe et al., "Amyloid PET in clinical practice: its place in the multidimensional space of Alzheimer's disease," NeuroImage Clin. 2(1), 497-511 (2013). 
Eravuchira et al.: Hollow core photonic crystal fiber-assisted Raman spectroscopy as a tool...

15. L. Mosconi et al., "Pre-clinical detection of Alzheimer's disease using FDG-PET, with or without amyloid imaging," J. Alzheimer's Dis. 20(3), 843-854 (2010).

16. X. Y. Zhang et al., "PET/MR imaging: new frontier in Alzheimer's disease and other dementias," Front. Mol. Neurosci. 10(Nov.), 1-12 (2017).

17. K. Blennow et al., "Clinical utility of cerebrospinal fluid biomarkers in the diagnosis of early Alzheimer's disease," Alzheimer's Dement. 11(1), 58-69 (2015).

18. T. Tapiola et al., "Cerebrospinal fluid $\beta$-amyloid 42 and tau proteins as biomarkers of Alzheimer-type pathologic changes in the brain," Arch. Neurol. 66(3), 382-389 (2009).

19. P. Gagni et al., "Development of a high-sensitivity immunoassay for amyloid-beta 1-42 using a silicon microarray platform," Biosens. Bioelectron. 47, 490-495 (2013).

20. P. Carneiro et al., "Alzheimer's disease: development of a sensitive label-free electrochemical immunosensor for detection of amyloid beta peptide," Sens. Actuators, B Chem. 239, 157-165 (2017).

21. J. V. Rushworth et al., "A label-free electrical impedimetric biosensor for the specific detection of Alzheimer's amyloid-beta oligomers," Biosens. Bioelectron. 56, 83-90 (2014).

22. S. Chanpimol et al., "A fluorescence assay for detecting amyloid- $\beta$ using the cytomegalovirus enhancer/promoter," J. Biol. Methods 33, 1-7 (2017).

23. A. J. Haes et al., "A localized surface plasmon resonance biosensor: first steps toward an assay for Alzheimer's disease," Nano Lett. 4(6), 1029-1034 (2004).

24. H. T. Beier et al., "Application of surface-enhanced Raman spectroscopy for detection of beta amyloid using nanoshells," Plasmonics 2(2), 55-64 (2007).

25. M. Vestergaard et al., "Detection of Alzheimer's tau protein using localised surface plasmon resonance-based immunochip," Talanta 74(4), 1038-1042 (2008).

26. J. Skoch et al., "Development of an optical approach for noninvasive imaging of Alzheimer's disease pathology," J. Biomed. Opt. 10(1), 011007 (2005).

27. O. Harbater and I. Gannot, "Fluorescent probes concentration estimation in vitro and ex vivo as a model for early detection of Alzheimer's disease," J. Biomed. Opt. 19(12), 127007 (2014).

28. R. Buividas et al., "Statistically quantified measurement of an Alzheimer's marker by surface-enhanced Raman scattering," J. Biophotonics 8(7), 567-574 (2015).

29. M. Wang and R. D. JiJi, "Spectroscopic detection of $\beta$-sheet structure in nascent $\mathrm{A} \beta$ oligomers," J. Biophotonics 4(9), 637-644 (2011).

30. G. Devitt et al., "Raman spectroscopy: an emerging tool in neurodegenerative disease research and diagnosis," ACS Chem. Neurosci. 9(3), 404-420 (2018).

31. C. Capitini et al., "Structural differences between toxic and nontoxic HypF-N oligomers," Chem. Commun. (Cambridge) 54(62), 8637-8640 (2018).

32. C. D'Andrea et al., "Nanoscale discrimination between toxic and nontoxic protein misfolded oligomers with tip-enhanced Raman spectroscopy," Small 14(36), 1800890 (2018).

33. I. H. Chou et al., "Nanofluidic biosensing for $\beta$-amyloid detection using surface enhanced Raman spectroscopy," Nano Lett. 8(6), 1729-1735 (2008).

34. I. Choi, Y. S. Huh, and D. Erickson, "Ultra-sensitive, label-free probing of the conformational characteristics of amyloid beta aggregates with a SERS active nanofluidic device," Microfluid. Nanofluid. 12(1-4), 663-669 (2012).

35. T. Demeritte et al., "Hybrid graphene oxide based plasmonic-magnetic multifunctional nanoplatform for selective separation and label-free identification of Alzheimer's disease biomarkers," ACS Appl. Mater. Interfaces 7(24), 13693-13700 (2015).

36. D. Yan et al., "Fiber enhanced Raman sensing of levofloxacin by PCF bandgap-shifting into the visible range," Anal. Methods 10(6), 586-592 (2018).

37. S. Hanf et al., "Fast and highly sensitive fiber-enhanced Raman spectroscopic monitoring of molecular $\mathrm{H} 2$ and $\mathrm{CH} 4$ for point-of-care diagnosis of malabsorption disorders in exhaled human breath," Anal. Chem. 87(2), 982-988 (2015).

38. S. Hanf et al., "Fiber-enhanced Raman multigas spectroscopy: a versatile tool for environmental gas sensing and breath analysis," Anal. Chem. 86(11), 5278-5285 (2014).

39. F. Eftekhari et al., "Examining metal nanoparticle surface chemistry using hollow-core, photonic-crystal, fiber-assisted SERS," Opt. Lett. 37(4), 680 (2012). 
40. X. Yang et al., "Direct molecule-specific glucose detection by Raman spectroscopy based on photonic crystal fiber," Anal. Bioanal. Chem. 402(2), 687-691 (2012).

41. A. Khetani et al., "Hollow core photonic crystal fiber for monitoring leukemia cells using surface enhanced Raman scattering (SERS)," Clin. Cancer Res. J. Z. Darzynkiewicz, H. Zhao, Cell Cycle Anal. by Flow Cytom. eLS 12(4), 2662-2669 (2006).

42. H. Yan et al., "Hollow core photonic crystal fiber surface-enhanced Raman probe hollow core photonic crystal fiber surface-enhanced Raman probe," Appl. Phys. Lett. 89, 204101 (2006).

43. J. Z. Zhang et al., "Hollow-core photonic crystal fibers for surface-enhanced Raman scattering probes," Int. J. Opt. 2011(10) (2011).

44. D. Yan et al., "Fiber enhanced Raman spectroscopic analysis as a novel method for diagnosis and monitoring of diseases related to hyperbilirubinemia and hyperbiliverdinemia," Analyst 141(21), 6104-6115 (2016).

45. A. V. Markin, N. E. Markina, and I. Y. Goryacheva, "Raman spectroscopy based analysis inside photonic-crystal fibers," Trends Anal. Chem. 88, 185-197 (2017).

46. K. K. Chow et al., "A Raman cell based on hollow core photonic crystal fiber for human breath analysis," Med. Phys. 41(9), 092701 (2014).

47. C. Pappas et al., "Evaluation of a Raman spectroscopic method for the determination of alcohol content in Greek spirit Tsipouro," Curr. Res. Nutr. Food Sci. J. 4(Special-IssueOctober), 1-9 (2016).

48. S. Hu, K. M. Smith, and T. G. Spiro, "Assignment of protoheme resonance Raman spectrum by Heme labeling in myoglobin," J. Am. Chem. Soc. 118(50), 12638-12646 (1996).

49. M. Banchelli et al., "Spot-on SERS detection of biomolecules with laser-patterned dot arrays of assembled silver nanowires," ChemNanoMat 5(8), 1036-1043 (2019).

50. Y. Kitahama and Y. Ozaki, "Surface-enhanced resonance Raman scattering of hemoproteins and those in complicated biological systems," Analyst 141(17), 5020-5036 (2016).

51. M. Banchelli et al., "Controlled veiling of silver nanocubes with graphene oxide for improved surface-enhanced Raman scattering detection," ACS Appl. Mater. Interfaces 8(4), 2628-2634 (2016).

52. P. Matteini et al., "Concave gold nanocube assemblies as nanotraps for surface-enhanced Raman scattering-based detection of proteins," Nanoscale 7(8), 3474-3480 (2015).

53. M. Banchelli et al., "Triggering molecular assembly at the mesoscale for advanced Raman detection of proteins in liquid," Sci. Rep. 8(1), 1-8 (2018).

54. H. L. Sun et al., "The correlations of plasma and cerebrospinal fluid amyloid-beta levels with platelet count in patients with Alzheimer's disease," Biomed Res. Int. 2018(1), 1-8 (2018).

55. K. B. N Andreasen et al., "Cerebrospinal fluid beta-amyloid(1-42) in Alzheimer disease: differences between early- and late-onset Alzheimer disease and stability during the course of disease," Arch. Neurol. 56(6), 673-680 (1999).

Biographies of the authors are not available. 